\title{
Influência da atividade física em grupo na qualidade de vida e variáveis cardiorrespiratórias em idosos institucionalizados
}

Influence of physical activity group in the quality of life and cardiorespiratory variables in institutionalized elderly

FisiSenectus. Unochapecó Ano 4, n. 1 - Jan/Jun. 2016 p. $22-31$

Ana Laura Nicoletti Carvalho Petry. ana.petry@uffs.edu.br

Doutora em Ciências pela Universidade de São Paulo (USP). Fisioterapeuta da Universidade Federal da Fronteira Sul (UFFS) Campus Passo Fundo/RS.

Diana Menegotto.dianamenegotto@hotmail.com

Fisioterapeuta graduada pela Universidade Regional Integrada do Alto Uruguai e das Missões (URI) - Campus Erechim/ RS.

Carla Spazzini Barro. carla_barro@hotmail.com

Fisioterapeuta graduada pela Universidade Regional Integrada do Alto Uruguai e das Missões (URI) Campus Erechim/ RS.

Naiana Muntini.nmuntini@gmail.com

Especialista em Fisioterapia Hospitalar pela Universidade de Passo Fundo (UPF). Fisioterapeuta graduada pela Universidade de Passo Fundo (UPF).

Daniele Rossato. danirossato@hotmail.com

Mestre em Neurociências pela Universidade Federal do Rio Grande do Sul (UFRGS).

Fisioterapeuta do Hospital de Clínicas de Porto Alegre/RS.

\section{Resumo}

Introdução: 0 envelhecimento populacional é um fenômeno relevante em todo mundo, sendo que atenção tem sido dada à qualidade de vida da população idosa. Objetivo: Avaliar os efeitos da atividade física sobre a qualidade de vida de idosos institucionalizados, identificar o perfil desses indivíduos e verificar a influência da atividade física sobre os sinais vitais. Materiais e métodos: A amostra foi composta por sete indivíduos, de ambos os sexos. O programa de exercícios foi realizado em uma frequência de três vezes semanais no período de um mês. A intervenção foi composta de exercícios de aquecimento, alongamentos, amplitude de movimento ativa, atividades lúdicas e relaxamento. A avaliação foi realizada por meio de um questionário de caracterização da amostra, bem como aplicação do questionário WHOQOL-OLD para avaliar a qualidade de vida, onde foi aplicado pré e pósintervenção. Os sinais vitais: PAS, PAD, FC, FR, Sap02 foram verificadas pré e pós-sessão. Resultados: Verificou-se neste estudo que os participantes apresentavam idade média de 70 anos, sendo a maior prevalência do sexo feminino (86\%) e de indivíduos não escolarizados (57\%). Em relação ao escore bruto pré e pós intervenção do questionário WHOQOL - OLD, observamos diferença estatisticamente significativa $(p<0,05)$ evidenciando uma melhora na

\section{Fisißenectus}


qualidade de vida desta população. Observamos uma redução estatisticamente significativa na PAD, PAS e Sp02 no momento pós-intervenção. Conclusão: Conclui-se assim que o programa de atividade física não proporcionou alterações relevantes clinicamente nos sinais vitais. Destaca-se ainda que a atividade física em grupo proporcionou uma melhora global da qualidade de vida dos idosos institucionalizados.

\title{
Palavras-chave
}

Envelhecimento; Qualidade de vida; Atividade motora.

\begin{abstract}
Introduction: Population aging is a relevant phenomenon worldwide, and attention has been paid to the quality of life of the elderly population. Objective: The present study aimed to evaluate the effects of physical activity on quality of life of elderly and institutionalized individuals near senility, identify the profile of these individuals and to investigate the influence of physical activity on vital signs. Materials and methods: The sample was composed of seven individuals of both sexes. The exercise program was conducted at a frequency of three times a week during one month. The intervention consisted of warm-up exercises, stretching, active range of motion, leisure activities and relaxation. The evaluation was conducted through a questionnaire sample characterization, and application of the WHOQOL -OLD to assess quality of life, which was applied pre -and post- intervention. Vital signs: SBP, DBP, HR, FR, oxigenation pre and post session were recorded. Results: It was found that participants in this study had a mean age of 70 years, with a higher prevalence of females (86\%) and non- educated individuals (57\%). Regarding the raw score pre and post intervention WHOQOL - OLD, a statistically significant difference $(p<0.05)$ indicating an improvement in the quality of life of this population. We observed a statistically significant reduction in DBP, SBP and oxigenation in the postintervention time. Conclusions: It follows therefore that the physical activity program provided no clinically relevant changes in vital signs. Note also that physical activity group gave an overall improvement in the quality of life of institutionalized elderly.
\end{abstract}

\section{Keywords}

Aging; Quality of life; Motor activity.

\section{Introdução}

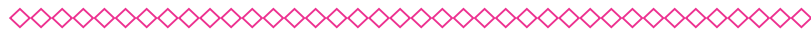

0 envelhecimento populacional é um fenômeno relativamente novo em todo mundo. Conforme dados do Censo Demográfico do Instituto Brasileiro de Geografia e Estatística (IBGE), há cerca de 10 milhões de pessoas na população brasileira com idade superior a 65 anos e no ano de 2020 o Brasil terá a sexta maior população mundial em números absolutos de idosos ${ }^{1}$.

Fisiologicamente, o envelhecimento tem início relativamente precoce logo após a fase de desenvolvimento e estabilização, ou seja, após a segunda década de vida, perdurando por longo período pouco perceptível, até que as alterações funcionais e estruturais tornem- se grosseiramente evidentes, geralmente após a quarta década de vida².
Sabe-se que o envelhecimento está associado a uma diminuição na prática de atividade física, com crescente prevalência de doenças crônico-degenerativas ${ }^{3}$. Paralelamente a esse processo, ocorre declínio das capacidades funcionais, diminuição da autonomia, gerando um maior comprometimento na qualidade de vida desta população ${ }^{1}$.

A prática de atividade física orientada e realizada regularmente aumenta a expectativa de vida, reduzindo as taxas de morbidade e mortalidade, assim como pode diminuir o número de medicamentos prescritos. Também proporciona efeitos benéficos em nível neuromuscular, metabólico e psicológico, resultando na manutenção da independência, em maior autonomia, mobilidade, redução de quedas, melhorando a autoimagem e a autoestima, estimulando o contato social e os prazeres pela vida ${ }^{4,5}$. 
A prática regular de atividade física, contudo, atua na redução de fatores de risco, apresenta efeitos positivos na qualidade de vida e previne 0 aparecimento de doenças crônico-degenerativas ${ }^{6}$.

Estudo com 150 idosos de ambos os sexos, que praticavam exercícios físicos em duas universidades públicas do sul do Brasil demonstraram que a participação nessas atividades pode ser um dos fatores da percepção positiva sobre a autoimagem e autoestima ${ }^{7}$. A melhora na qualidade de vida proporcionada pela atividade física também está fortemente relacionada ao aspecto físico proporcionando a prevenção ou redução da pressão arterial, previne o ganho de peso ponderal, auxiliar na prevenção e redução da osteoporose, reduzindo também a ansiedade, a depressão e o estresse ${ }^{7}$.

Apesar de os benefícios da prática de atividade física ser amplamente divulgados, poucos são os que realizam tais atividades de forma regular e controlada no ambiente de institucionalização. As pesquisas que avaliam os efeitos da atividade física na qualidade de vida na população de idosos no Brasil estão crescendo, mas ainda são insuficientes diante do visível aumento desta população. Dentro desse contexto, estudos que busquem atualizar o conhecimento sobre a problemática do envelhecimento contribuem para uma formação de profissionais competentes no cuidado com o idoso, bem como incentivem os mesmos à implementação de ações voltadas para a promoção da saúde ${ }^{8}$.

Estimular e implementar a autonomia funcional na população idosa é um dos objetivos mais comuns aos profissionais da área da fisioterapia, visto que torná-los mais independentes é uma ação de fundamental relevância para a sociedade atual. Contudo, não basta abordar apenas o fator físico, pois existe uma inter-relação de vários fatores que culminam em um estilo de vida saudável, e por consequente uma qualidade de vida satisfatória.

Em virtude disso, este estudo teve por objetivo analisar a influência da atividade física em grupo sobre a qualidade de vida e variáveis cardiorrespiratórias de idosos institucionalizados no Lar dos Velhinhos - Sociedade Beneficente Jacinto Godoy, da cidade de Erechim (RS).

\section{Materiais e métodos}

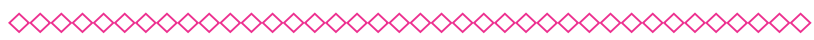

0 presente estudo caracterizou-se por apresentar caráter quantitativo do tipo experimento não controlado. A amostra foi composta de sete indivíduos, de ambos os sexos, selecionados de forma aleatória institucionalizados em uma instituição de longa permanência da cidade de Erechim/RS

Foram incluídos no estudo indivíduos que tivessem idade superior a 60 anos, institucionalizados, com indicação e liberação médica para a prática de atividades físicas e indivíduos sem distúrbios músculo-esqueléticos e osteoarticulares incapacitantes para a realização de atividade física. Foram excluídos de estudo indivíduos que tivessem algum distúrbio mental ou outra patologia incapacitante da pratica de atividades físicas e indivíduos que não tivessem participado de todas as sessões de atividades.

Inicialmente, solicitou-se autorização à instituição para a realização do estudo. Após, conversou-se com o médico responsável pelos indivíduos para seleção da amostra, uma vez que o médico indicou quais indivíduos estavam liberados para a prática das atividades físicas propostas nesta intervenção. Assim, a pesquisadora entrou em contato com os participantes selecionados onde explicou individualmente o estudo, os sujeitos que concordaram em participar assinaram o Termo de Consentimento Livre e Esclarecido.

No momento do contato inicial foram aplicados individualmente dois questionários: o primeiro contendo questões de identificação dos participantes, para identificação do perfil da amostra do estudo; o segundo para avaliação da qualidade de vida, o questionário WHOQOL-OLD, que é específico e validado no Brasil para idosos, sendo composto por 24 questões que avaliam seis facetas: Funcionamento Sensório; Autonomia; Atividades Passadas, Presentes e Futuras; Participação Social; Morte e Morrer e Intimidade ${ }^{9}$.

As atividades físicas foram realizadas em grupo, em local específico previamente determinado pela direção da instituição, durante 45 minutos, três vezes por semana, no período de um mês, totalizando 10 sessões. 0 protocolo de atividades físicas continha: 
a) Exercícios de alongamento muscular: os exercícios de alongamento foram realizados por pelo menos 10 minutos com 40 segundos para cada alongamento incluindo exercícios para a região cervical; região peitoral; flexores e extensores do punho; alongamento para membros superiores; alongamento para o tronco com flexão lateral, rotação de tronco e inclinação lateral; alongamento de membros inferiores (quadríceps e isquiotibiais);

b) Exercícios ativos: os exercícios ativos foram realizados por um período de aproximadamente 30 minutos e compreenderam exercícios ativos e/ ou ativos-assistidos de todos os grupos musculares (com 10 séries de cada) utilizando também faixas elásticas e bastões, exercícios lúdicos variados e exercícios respiratórios (exercício diafragmático, exercício de freno labial, exercício de inspiração profunda);

c) Relaxamento muscular: os exercícios de relaxamento foram realizados por aproximadamente 5 minutos e consistiam em atividades relaxantes como automassagem, música relaxante e exercícios respiratórios.

A pressão arterial sistólica (PAS) e diastólica (PAD) dos indivíduos, assim como a frequência cardíaca (FC), frequência respiratória (FR) e a saturação periférica de oxigênio (SpO2) foram verificadas antes e após cada sessão de intervenção fisioterapêutica. As aferições após as sessões de intervenção foram realizadas imediatamente após o término da atividade proposta.

Ao término do programa de atividades físicas, pós-intervenção, foi reaplicado aos participantes novamente da mesma forma o questionário para avaliação da qualidade de vida WHOQOL-OLD.

Os dados quantitativos foram analisados por meio da comparação dos valores dos escores individuais e total das facetas; e sinais vitais nos momentos pré e pós-intervenção fisioterapêutica. Foi utilizando o teste t Student pareado (dados paramétricos) para os escores do WHOQOL-OLD e para a $\mathrm{FC}$ e o teste de Wilcoxon (dados não-paramétricos) para FR, PAS, PAD e Sp02. Os testes foram executados com auxílio do software SPSS 15.0 (SPSS Inc., Chicago, ILL, USA). O valor de significância foi estabelecido em $p<0,05$ para todas as análises.
0 presente trabalho seguiu os princípios éticos contidos na Declaração de Helsique, sendo aprovado pelo Comitê de Ética em pesquisa da URI Campus de Erechim/RS sob número 117/TCH/11.

\section{Resultados}

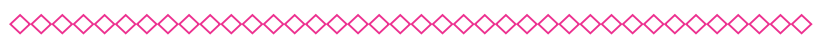

A amostra desta pesquisa foi composta por sete indivíduos. Inicialmente, 11 pacientes participaram das atividades propostas, no entanto, quatro indivíduos desistiram de participar da pesquisa na primeira semana de atividades, por motivos pessoais.

Com relação às características da amostra (Tabela 1), observou-se $86 \%$ do sexo feminino, a média de idade foi de 70,86 anos. A maioria da amostra foi constituída de indivíduos não escolarizados (57\%). Os participantes exerciam atividades profissionais (antes da institucionalização) com nível de exigência de baixa escolaridade. Quarenta e três por cento dos indivíduos eram solteiros, 29\% viúvos, $14 \%$ casados e $14 \%$ separados. Ao serem perguntados sobre a prática de atividade física, 57\% dos participantes elegeram a dança com atividade.

Considerando a análise dos sinais vitais (Tabela 2$)$, houve redução estatisticamente significativa nos valores de PAS $(p=0,003), \operatorname{PAD}(p=0,002)$ e SpO2 $(p=0,045)$ após a sessão de atividade física quando comparados com o momento pré atividade. Os valores de FC e FR não apresentaram diferença estatisticamente significante do momento pós-atividade em relação ao pré, no entanto, verificou-se discreta elevação dos valores de FC e redução dos valores da FR.

A Tabela 3 nos permite analisar os escores individuais das facetas do questionário de qualidade de vida WHOQOL-OLD. Verificou-se um aumento nos escores pós em todas as facetas, porém sem diferenças significativas. No entanto, ao avaliar os escores brutos totais, houve diferença estatisticamente significante $(p=0,007)$ no momento pós-intervenção comparado ao pré-intervenção.

\section{Discussão}

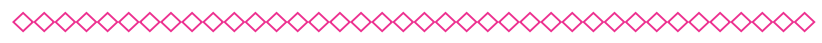

Estudos vêm demonstrando que o sexo feminino possui um padrão de vida mais ativo em relação 
ao masculino, tanto pelo envolvimento em atividades domésticas e sociais, como pela busca de atividades físicas e lazer ${ }^{10}$. Guimarães et al. ${ }^{11}$, ao realizar um estudo com 102 idosos pertencentes a um programa de atividade física em grupo, observou a predominância do sexo feminino em $82 \%$ dos participantes. Estes resultados assemelham-se aos encontrados por Marin et al. ${ }^{12}$, ao analisarem a realidade do idoso institucionalizado, na qual as mulheres representavam $64,9 \%$ dos idosos institucionalizados e os homens $35,1 \%$.

Resultado semelhante aos dessa pesquisa com relação à idade foram encontrados por Guedes e Silveira ${ }^{13}$, que analisaram a capacidade funcional de idosos institucionalizados da cidade de Passo Fundo (RS), e encontraram uma média de idade de 74,17 anos.

Na presente pesquisa observou-se uma maior prevalência de indivíduos não escolarizados $57 \%$. Davim et al. ${ }^{14}$, também encontraram alto índice de analfabetismo entre os idosos institucionalizados na cidade de Natal, onde, de três instituições pesquisadas, $46 \%$ do total dos idosos institucionalizados não eram alfabetizados. Vieira ${ }^{15}$ ao analisar instituições filantrópicas e particulares de Florianópolis, encontrou uma prevalência do grupo de analfabetos, cerca de $62 \%$ da população.

Todaro ${ }^{16}$ encontrou uma prevalência de indivíduos separados $47 \%$, seguidos por solteiros $40 \%$, casados $8 \%$ e viúvos $5 \%$. No entanto, resultados diferentes aos encontrados forma observados em um programa de fisioterapia em idosas não institucionalizadas e verificou-se que $55 \%$ eram casadas, $40 \%$ viúvas e $5 \%$ solteiras $^{17}$.

Em idosos institucionalizados em uma instituição de longa permanência na cidade de São Paulo observou-se que apenas 35\% dos participantes praticavam algum tipo de atividade física. Atividade de dança causa melhoras no bem-estar psicológico dos idosos, diminuindo os escores de tensão e depressão, além de promover melhora na agilidade, equilíbrio e aumento da flexibilidade ${ }^{17}$.

No indivíduo idoso se observa, que durante uma atividade física, quanto mais se eleva a intensidade do exercício, mais a FC tende a aumentar para buscar compensar o gasto energético gerado pelo exercício, visto que devido a um processo natural do envelhecimento o idoso apresenta diminuição na força contrátil do músculo cardíaco. Consequentemente haverá uma diminuição no débito cardíaco (DC), débito este que está intimamente ligado a alterações na $\mathrm{FC}^{18}$. Farinati e $\mathrm{Assis}^{19}$, ao estudarem a influência de exercícios aeróbicos sobre a FC, em 18 indivíduos, observaram aumento gradativo da FC, de acordo com os exercícios realizados, em relação ao repouso. Outro estudo analisou a influência da atividade física nos sinais vitais, em solo e água, em 22 idosos. Foi observado um aumento significativo da FC e PA após a realização das atividades, sem diferença estatística entre 0 meio realizado 20 .

Nesta pesquisa, observou-se um aumento na FR após o programa de atividades, porém sem diferenças significativas. Aumentos na FR são esperados, visto que há necessidade de se respirar rápido e profundamente para introduzir oxigênio no organismo e remover o excesso de gás carbônico produzido pelas células ${ }^{21}$.

Estudos epidemiológicos e clínicos têm demonstrado efeitos benéficos da prática de atividade física sobre a pressão arterial de indivíduos de todas as idades. Alto nível de atividade física diária está associado a menores níveis de pressão arterial em repouso. A prática regular de exercício físico tem demonstrado prevenir o aumento da pressão arterial associado à idade, mesmo em indivíduos com risco aumentado de desenvolvê-la ${ }^{22}$. Verificou-se, neste trabalho uma redução de 4 a 5 $\mathrm{mmHg}$ nas pressões arteriais sistólicas e diastólicas. 0 período observado foi curto e especula-se que efeitos mais proeminentes poderiam ocorrer em relação a PA com a continuidade do programa de exercícios propostos.

Redução estatisticamente significante na saturação de oxigênio foi encontrada neste trabalho, no entanto todos os valores no pré e pós-intervenção permaneceram dentro dos valores de normalidade para pacientes idosos considerados acima de $90 \%$ a $92 \%$. Portanto, as atividades físicas propostas neste protocolo não causaram prejuízo na oxigenação sanguínea.

A atividade física, além dos benefícios físicos, tem um papel importante na qualidade de vida, que engloba domínios como capacidade funcional, estado emocional, interação social, atividade intelectual, autocuidado, suporte familiar, estado de 
saúde, valores culturais, éticos, religiosos, estilo de vida, satisfação com o emprego ou com atividades diárias e ambiente em que se vive. Está relacionada à autoestima e ao bem-estar individual ${ }^{23}$.

Verificou-se uma boa qualidade de vida nesta população, determinados pela soma dos escores dos domínios do questionário WHOQOL-OLD, visto que a soma dos escores, no momento pós-intervenção, é de $93,28 \pm 5,74$, em um total de 120 pontos possíveis. Ao realizar um estudo avaliando a qualidade de vida de mulheres idosas, utilizando o questionário WHOQOL-OLD, obteve um escore total de qualidade de vida de $66,9 \pm 11,7$, inferior ao obtido neste estudo. Viver mais e melhor liga-se às principais aspirações dos idosos neste novo cenário do mundo contemporâneo. Alguns aspectos são considerados importantes para dizer se um indivíduo possui ou não boa qualidade de vida: os aspectos sociais, a atividade física, o seu comportamento preventivo, sua percepção de bem-estar e percepção subjetiva de saúde, entre outros. Para tanto, um apanhado desses valores pode ser considerado como indicadores da percepção da qualidade de vida do próprio individuo ${ }^{24}$.

Um programa de atividade física para os idosos favorece a melhoria da aptidão física, do domínio corporal, da autoestima e da capacidade funcional, além de ampliar as relações sociais, diminuindo a chance de aparecimento de doenças psicossociais como a depressão ${ }^{25}$. Em uma revisão de literatura, autores observaram que a depressão representa enfermidade mental mais frequente no idoso, comprometendo de forma significativa sua qualidade de vida. Entretanto, quando o idoso está engajado em um programa de atividade física, regular e bem planejado, isso contribui para a minimização do sofrimento psíquico do idoso deprimido, além de oferecer oportunidade de envolvimento psicossocial, elevação da autoestima e implementação das funções cognitivas, fatores estes muito importantes no contexto dessa população ${ }^{26}$. Em estudo sobre o nível de qualidade de vida em 50 idosas praticantes de danças e musculação, utilizando o mesmo instrumento de avaliação do presente estudo, observou que a atividade proposta apresentou melhora significativa na qualidade de vida do grupo intervenção, em relação ao grupo controle 27 .
Segundo Resnick ${ }^{28}$, o exercício físico regular pode melhorar a saúde, facilitar e promover o contato social, e favoravelmente afetar a qualidade de vida, levando à melhoria da vida social e interação, exercício físico regular motiva as pessoas a adotarem melhores hábitos de estilo de vida e é uma alternativa de tratamento para estresse, o que tem impactos favoráveis sobre todos os aspectos da vida. Talvez seja a soma total dessas alterações, o que melhorou a qualidade de vida de nossos voluntários institucionalizados.

0 presente trabalho apresenta algumas limitações, o que permite algumas reflexões tais como: (1) o número pequeno da amostra (inicialmente eram 11 participantes e ao final permaneceram sete indivíduos) e o número de sessões limita algumas conclusões sobre o protocolo proposto, principalmente em relação aos resultados encontrados, que podem não ser reprodutíveis em amostras com número de participante maiores, porém, entende-se que em instituições de longa permanência para idosos é difícil a aderência a protocolos de pesquisa, pois a grande maioria dos idosos possui limitações ortopédica e/ou cognitivas para a prática controlada de atividade física; (2) mesmo este estudo não contendo um grupo controle o objetivo foi analisar os possíveis ganhos ou prejuízos do indivíduo em relação a ele mesmo antes e após o protocolo; (3) embora a maioria dos idosos praticasse dança (no momento de aplicação da pesquisa eles não tiveram as aulas em virtude de ser período de férias), essa não era uma atividade controlada com protocolo específico.

\section{Considerações finais}

$\infty \times \infty \times \infty \times \infty \times \infty \times \infty \times \infty \times \infty \times \infty \times \infty \times \infty \infty \infty$

Conclui-se, assim, que o protocolo proposto melhorou a qualidade de vida geral trazendo benefícios ao grupo de idosos institucionalizados no Lar dos Velhinhos em Erechim (RS). 0 comportamento estável e dentro da normalidade dos sinais vitais FC, FR, PA e Sp02, demonstrou segurança dos exercícios propostos e benefícios discretos sobre a pressão arterial.

Estudos que possam ter uma duração maior em número de sessões e com uma amostra maior, seriam de grande valia, possibilitando uma conclusão 
mais completa e mais específica sobre a influência da atividade física na qualidade de vida de indivíduos idosos institucionalizados.

\section{Referências}

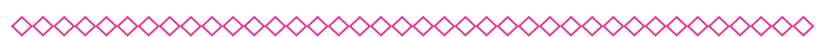

1. Januário RSB, Junior HS, Liutti MC, Decker D, Molari M. Qualidade de vida em idosos ativos e sedentários. ConScientiae Saúde. 2011;10(1):112-21.

2. Duarte YA, Diogo MJ. Atendimento domiciliar: um enfoque gerontológico. Rio de Janeiro: Atheneu; 2000

3. Scher, LML, Nobre F, Lima NKC. O papel do exercício físico na pressão arterial em idosos. Rev Bras Hipertens. 2008;15(4):228-231.

4. Shephard RJ. Envelhecimento, atividade física e saúde. São Paulo: Phorte; 2003.

5. Siqueira FV, Facchini LA, Hallal PC. Epidemiology of physiotherapy utilization among adults and elderly. Rev Saúde Públ. 2005;39(4):662-8.

6. Ferreira Filho C, Meneghini A, Riera ARP, Serpa Neto A, Teixeira GK, Ferreira C. Benefícios do exercício físico na hipertensão arterial sistêmica. Arq Méd ABC. 2007;32:82-7.

7. Maciel MG. Atividade física e funcionalidade do idoso. Motriz. 2011;16(4):1024-32.

8. Alencar NA, Souza Júnior, Aragão JCB, Ferreira MA, Dantas E. Nível de atividade física, autonomia funcional e qualidade de vida em idosas ativas e sedentárias. Fisioter Mov. 2010;23(3):473-81.

9. Fleck MP, Chachamovich E, Trentini C. Desenvolvimento e validação da versão em Português do módulo WHOQOL-OLD. Rev Saúde Públ. 2006;40(5):785-91.

10. Cousins SO, Keating N. Life cycle patterned on physical activity among sedentary and active older women. J Aging Phys Act. 1995;4(3):340-59.

11. Guimarães ACA, Mazo GZ, Simas JPN, Salin MS, Schwertner DS, Soares A. Idosos praticantes de atividade física: tendência a estado depressivo e capacidade funcional. Efdeportes. 2006;10(94).

12. Marin MJS,Miranda FA, Fabbri D, Tinelli LP, Storniolo LV. Compreendendo a história de vida de idosos institucionalizados. Rev Bras Geriatr Gerontol. 2012;15(1):147-54.

13. Guedes JM, Silveira RCR. Análise da capacidade funcional da população geriátrica institucionalizada na cidade de Passo Fundo- RS. Rev Bras Ciên Envelh Hum. 2004;10(21):10-21.

14. Davin RMB, Torres GV, Dantas SMM, Lima VM. Estudo com idosos de instituições asilares no município de Natal/RN: características socioeconômicas e de saúde. Rev Latino-Am Enfermage. 2004;12(3):518-24.

15. Vieira GF. Estudos dos parâmetros motores de idosos residentes em instituições asilares filantrópicas e particulares da grande Florianópolis [dissertação]. Florianópolis: Universidade do Estado de Santa Catarina; 2004.

16. Todaro MA. Dança: uma interação entre o corpo e a alma dos idosos. [dissertation]. Campinas: Universidade Estadual de Campinas; 2001.

17. Petyk J, Guedes JM, Sebben V. Programa de fisioterapia em idosas. Perspectiva. 2011;35(129):103-12.

18. Williams MA, Haskell WL, Ades PA, Amsterdam EA, Bittner V, Franklin BA, et al. Resistance exercise in individuals with and without cardiovascular disease: 2007 update: a scientific statement from the American Heart Association Council on Clinical Cardiology and Council on Nutrition, Physical Activity, and Metabolism. Circ. 2007;116(5):572-84.

19. Farinatti PTV, Assis BFCB. Estudo da freqüência cardíaca, pressão arterial e duploproduto em exercícios contra-resistência e aeróbico contínuo. Rev Bras Ativ Física Saúde 2000;5:5-16.

20. Junior JA, Lima JP, Oliveira MM. Estudo comparativo da frequência cardíaca e da pressão arterial antes e após exercício aeróbico 
no solo e na água em idosos do "projeto vida ativa na terceira idade" [dissertação]. Manaus: Universidade da Amazônia; 2007.

21. Barroso WKS, Jardim PCBV, Vitorino $P V$, Bittencourt A, Miquetichuc F. Influência da atividade física programada na pressão arterial de idosos hipertensos sob tratamento não-farmacológico. Rev Assoc Med Bras. 2008;54(4):328-33.

22. Ciolac EG, Guimaraes GV. Exercício físico e síndrome metabólica. Rev Bras Med Esporte. 2004;10(4):319-324.

23. Carvalho ED, Valadares ALR, Costa-Paiva LH, Pedro AO, Morais SS, Pinto-Neto AM. Atividade física e qualidade de vida em mulheres com 60 anos ou mais: fatores associados. Rev Bras Ginecol Obstet. 2010;32(9):433-40.

24. Amaro LE, Johann JR, Meira PR. O estilo de vida do idoso urbano: o caso Porto Alegre - RS. Rev Bras Ciên Envelh Hum. 2007;4(1):115-126.
25. Borges GF. Nível de atividade física, capacidade funcional e qualidade de sono de idosas [dissertação]. Florianópolis: Universidade Federal de Santa Catarina; 2006.

26. Stella F, Gobbi S, Corazza DI, Costa JLR. Depressão no idoso: diagnóstico, tratamento e benefícios da atividade física. Motriz. 2002;8(3):91-8.

27. Castro JC, Bastos FAC, Cruz THP, Giani TS, Ferreira MA, Dantas EHM. Níveis de qualidade de vida em idosas ativas praticantes de dança, musculação e meditação. Rev Bras Geriatr Gerontol. 2009;12(2):255-65.

28. Resnick B. Uma abordagem de sete passos para iniciar um programa de exercícios para idosos. Rev Bras Psiquiatr. 2000;39:243-52. 


\section{Anexos}

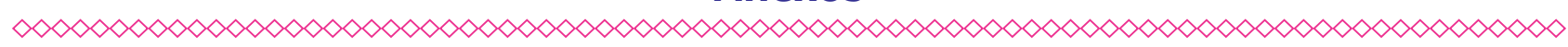

Tabela 1 - Dados referentes à caracterização da amostra (n=7), 2016

\begin{tabular}{lc} 
Variáveis & Resultados \\
Sexo (\%) & $14 \%$ \\
Masculino & $86 \%$ \\
Feminino & 70,86 \\
Idade (anos, média) & \\
Escolaridade (\%) & $57 \%$ \\
Não escolarizado & $29 \%$ \\
Ensino Fundamental Incompleto & $14 \%$ \\
Ensino Fundamental Completo & \\
Profissão (\%) & $43 \%$ \\
Dona de casa & $14 \%$ \\
Agricultora & $14 \%$ \\
Marceneiro & $14 \%$ \\
Costureira & $14 \%$ \\
Cozinheira & \\
Estado civil (\%) & $43 \%$ \\
Solteiro & $14 \%$ \\
Casado & $14 \%$ \\
Separado & \\
Atividade física (\%) & $57 \%$ \\
Praticam & $43 \%$ \\
Não praticam & \\
Tipo de atividade física praticada (\%) & $57 \%$ \\
\hline Dança & $(14 \%$ \\
\hline
\end{tabular}

Fonte: Elaborado pelos autores

(clique para voltar ao texto)

Tabela 2 - Dados referentes aos sinais vitais nos momentos pré e pós-atividade física, 2016

\begin{tabular}{lcc} 
Variáveis & Pré-intervenção $^{\text {a }}$ & Pós-intervenção $^{\text {a }}$ \\
FC (bpm) & $80,08 \pm 8,66$ & $80,55 \pm 7,20$ \\
FR (rpm) & $21,65 \pm 2,52$ & $22,06 \pm 1,87$ \\
PAS (mmHg) & $134,91, \pm 21,11$ & $129,86 \pm 15,74^{\text {b }}$ \\
PAD (mmHg) & $86,71 \pm 12,24$ & $82,14 \pm 12,50^{\mathrm{b}}$ \\
SpO2 (\%) & $96,19 \pm 2,54$ & $95,86 \pm 2,10^{\mathrm{b}}$ \\
\hline
\end{tabular}

aValores expressos em média \pm desviopadrão.

${ }^{\mathrm{b}} \boldsymbol{p}<0,05$ em relação ao pré intervenção.

Fonte: Elaborado pelos Autores

(clique para voltar ao texto) 
Tabela 3 - Dados referentes ao questionário de qualidade de vida WHOQOL-OLD nos momentos pré e pós-intervenção fisioterapêutica, 2016

\begin{tabular}{lcc} 
Facetas & Escore préa $^{\mathrm{a}}$ & Escore pós $^{\mathrm{a}}$ \\
1- Funcionamento dos & $16,14 \pm 2,97$ & $16,71 \pm 2,36$ \\
sentidos & & \\
2- Autonomia & $12,71 \pm 1,11$ & $14 \pm 2,08$ \\
3- Atividades presentes & $14,4 \pm 2,61$ & $15 \pm 2,08$ \\
passadas e futuras & & \\
4- Participação social & $15,29 \pm 1,50$ & $15,43 \pm 0,98$ \\
5- Morte e morrer & $15,42 \pm 3,69$ & $17,57 \pm 1,62$ \\
6- Intimidade & $13,86 \pm 1,46$ & $14,57 \pm 1,27$ \\
Escore total: & $87,57 \pm 6,29$ & $93,28 \pm 5,744^{\text {b }}$ \\
\hline
\end{tabular}

aValores expressos em média \pm desvio padrão.

${ }^{\mathrm{b}} \boldsymbol{p}<0,05$ em relação ao escore pré.

Fonte: Elaborado pelos autores

(clique para voltar ao texto) 